\title{
Combination of Roadside and In-Vehicle Sensors for Extensive Visibility Range Monitoring
}

\author{
Nicolas Hautière \\ Université Paris-Est \\ LEPSIS, INRETS/LCPC \\ 58 boulevard lefebvre, 75015 Paris, France \\ hautiere@lcpc.fr
}

\author{
Abderrahmane Boubezoul \\ Université Paris-Est \\ LEPSIS, INRETS/LCPC \\ 58 boulevard lefebvre, 75015 Paris, France \\ boubezou@lcpc.fr
}

\begin{abstract}
Fog is a local meteorological phenomena which drastically reduces the visibility range. Fog detection and visibility range estimation are critical tasks for road operators who need to warn the drivers and advise them on speed reductions. To achieve this task, fixed sensors are quite accurate but they have a reduced spatial cover. Mobile sensors are less accurate, but they have a good spatial cover. Based on the combination of roadside sensors and in-vehicle devices (sensors or fog lamps), a data fusion framework is presented aiming at taking the advantages of both fixed and mobile sensors for the extensive detection and estimation of the fog density. The proposed solution is implemented by means of a local dynamic map fed by vehicle to infrastructure (V2I) communication, which gives a coherent view of the road environment.
\end{abstract}

Keywords-fog detection; visibility range; data fusion; uncertainty; local dynamic map; (V2I) communication.

\section{INTRODUCTION}

The presence of dense fog on a road network affects the safety and may trigger reductions of the mandatory speeds. For example, a mandatory speed of $50 \mathrm{~km} / \mathrm{h}$ should be triggered if the visibility is below $50 \mathrm{~m}$. Unfortunately, meteorological centers are not able to monitor fog areas precisely since fog is a local phenomena. Road operators need to deploy dedicated sensors. These are expensive however and, sensitive to the inhomogeneity of fog as well. To improve fog detection, camera-based approaches are being developed since the camera has become a wide-spread low cost technology [5], [9]-[11], [15]. The accuracy of fixed camera-based sensors depends on the resolution and mounting height, and can be quite good. However, they cover a limited area. Another approach consists in using the sensors or the fog lamps which are equipped in the vehicles [3], [13], [14]. The quality of information brought from such devices is lower, but the area covered is obviously bigger. Fusing roadside and in-vehicle data sources comes as a natural solution to get a more extensive and more accurate estimation of the visibility range in foggy weather.

Thanks to the recent development of wireless communication between vehicles and infrastructure, the implementation of such data fusion is now possible. For instance, the
SAFESPOT project is developing a comprehensive architecture based on Vehicle to Vehicle (V2V) and Vehicle to Infrastructure (V2I) communications [4]. In particular, the so-called Local Dynamic Map (LDM) containing outputs from all the sensors in a single spatio-temporal database allows designing high level data fusion modules [2]. In this paper, we present our sensor combination framework dedicated to visibility range monitoring. We first present the architecture of the SAFESPOT project, in particular the road-side unit (RSU) and the LDM. Second, we recall the notion of meteorological visibility, on which our work is based. Then, we present the different data sources we have at our disposal for meteorological visibility, in particular the camera-based approach we developed. Third, we present our data fusion model and demonstrate its use on experimental data.

\section{ThE SAFESPOT INTEGRATED PROJECT}

\section{A. General Description}

By combining data from vehicle-side and road-side sensors, the SAFESPOT project aims at extending the time in which an accident is forecasted from the range of milliseconds up to seconds thanks to V2V and V2I communication. The system is based on communicating onboard units and road-side units which share a similar architecture. Hazard \& Incident Warning (H\&IW) and Speed Alert (SpA) applications triggered by degraded weather conditions are among the foreseen infrastructure-based SAFESPOT applications.

\section{B. The SAFESPOT Infrastructure Platform}

The primary functions of the SAFESPOT RSU are data acquisition, processing and storage. The data input come from several different sources. The most important are the roadside sensors but also the SAFESPOT vehicles. To improve the quality of information provided by the different inputs, the RSU performs three levels of processing. Preprocessing transforms raw sensor data into information useful for data fusion. Object Refinement (OR) merges data from different sources in order to improve the confidence of detection of moving objects, to extend the knowledge 


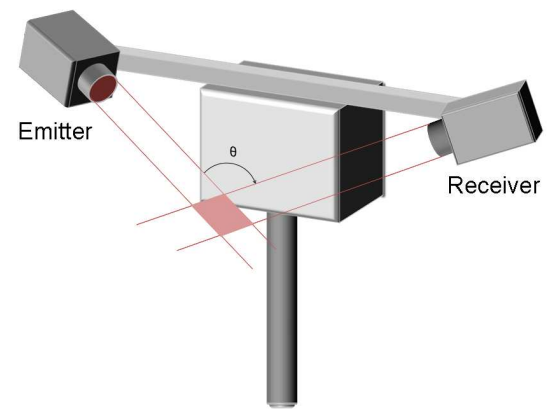

Figure 1. Diagram of a road visibilitymeter.

associated with objects and to locate them by means of map matching algorithms. Situation Refinement (SR) merges data describing traffic situations such as congestion, road weather or other black spots. In contrast to the object refinement, incoming data concerning a particular situation is merged with an unambiguous reference to the road map. The final results of the data fusion are written into a dedicated Local Dynamic Map (LDM). The function of the so-called 'Environmental Consolidator' (one SR module), presented in this paper, is to deal with the weather conditions.

\section{Meteorological VisibiLity}

\section{A. Definition}

Fog is thick cloud of microscopic water droplets suspended at ground level. When light propagating in fog encounters a droplet, the luminous flux is scattered in all directions. The amount of energy that is lost along the way is described by the extinction coefficient $k$. It depends on the droplet size distribution and the concentration. The proportion of energy transmitted between two points in fog is known as the transmissivity $T$ and decreases exponentially with distance $d$ (Beer Lambert's law):

$$
T=e^{-k d}
$$

The main effect of light scattering in the presence of fog is an overall reduction of contrasts as a function of distance. This effect is generally described by the meteorological visibility $V_{m e t}$, defined as the greatest distance at which a black object can be recognized in the sky against the horizon [6]. Using (1) with a contrast threshold of 5\% yields the following approximate relation between $V_{m e t}$ and the extinction coefficient $k$ :

$$
V_{\text {met }}=-\log (0.05) / k \approx 3 / k
$$

\section{B. Road Meteorology}

According to [1], road visibility is defined as the horizontal visibility for a driver whose eyes are $1.2 \mathrm{~m}$ above the roadway. It may be reduced to less than $400 \mathrm{~m}$ by fog, precipitations or projections. The standard classes of visibility range for road applications are <50, 50-100, 100200 and 200-400.

\section{Data Sources for Meteorological Visibility}

\section{A. Roadside Sensors}

1) Road Visibilitymeters: These systems were developed for road applications, primarily for conducting measurements under conditions of thick fog. They enable quantifying the light scattered within a sufficiently wide and well-defined solid angle [12]. In order to carry out such measurements, a light beam is concentrated onto a small volume of air (see Fig. 1). The proportion of light being scattered toward the receiver would then be:

$$
I=A I_{0} V f(\vartheta) e^{-k d}
$$

with $I$ the intensity scattered in the direction of the receiver, $A$ a constant dependent on power and source optics, $I_{0}$ the source intensity, $V$ the scattering volume, $f(\vartheta)$ the phase function, $\vartheta$ the scattering direction, $k$ the extinction coefficient and $d$ the length of the optical path between emitter and receiver. Generally speaking, the optical path $d$ is small and the transmission factor $e^{-k d}$ is assimilated to 1 and $f(\vartheta)$ is proportional to $k$. (3) thereby becomes:

$$
k=\frac{1}{A^{\prime}} \frac{I}{I_{0}}
$$

where $A^{\prime}$ designates a constant that depends on device characteristics. According to [7], the accuracy of such sensors is about $+/-10-20 \%$ over the field range. On the other hand, the small size of the scattering volume makes measurements highly sensitive to non-homogeneities in the fog. Moreover, such sensors cannot run other applications, contrary to a video-surveillance system. This is the topic of the next section.

2) Road-Side Camera: The apparent luminance of the road pavement $L$ is given by Koschmieder's law [16] which adds to (1) a second term corresponding to the atmospheric veil:

$$
L=L_{0} e^{-k d}+L_{f}\left(1-e^{-k d}\right)
$$

where $L_{0}$ denotes the intrinsic luminance of the pavement and $L_{f}$ the atmospheric luminance.

Assuming that the road is locally planar, the distance of a point located at the range $d$ on the roadway can be expressed in the image plane, assuming a pinehole camera model, by:

$$
d=\lambda /\left(v-v_{h}\right)
$$

where $\lambda=\frac{H \alpha}{\cos (\theta)}$ and $v_{h}=v_{0}-\alpha \tan (\theta) . \theta$ denotes the pitch angle of the camera, while $v_{h}$ represents the vertical position of the horizon line (see Fig. 2). The intrinsic parameters of the camera are its focal length $f$, and the size $t_{p}$ of a pixel. We have also made use herein of $\alpha=\frac{f}{t_{p}}$. $H$ denotes the sensor mounting height. In a foggy image, the intensity $I$ of a pixel is the result of the camera response function crf applied to (5). Assuming that crf is linear, (5) becomes:

$$
I=\operatorname{crf}(L)=R e^{-k d}+A_{\infty}\left(1-e^{-k d}\right)
$$




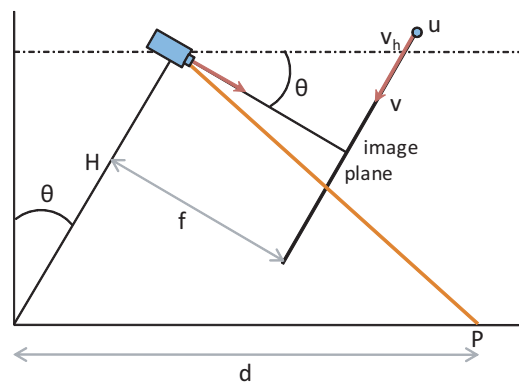

Figure 2. Modeling of the camera within the road environment. $v_{h}$ : image line corresponding to the horizon line in the image.

where $R$ is the intrinsic intensity of the pixel, i.e. the intensity corresponding to the intrinsic luminance value of the corresponding scene point and $A_{\infty}$ is the background sky intensity. After a change of $d$ according to $v$ (6), one obtains the following by taking the second derivative of $I$ with respect to $v$ :

$$
\partial^{2} I / \partial v^{2}=0 \Longleftrightarrow k=2\left(v_{i}-v_{h}\right) / \lambda
$$

where $v_{i}$ denotes the position of the inflection point of $I(v)$. $V_{\text {met }}$ is deduced using (2). To be able to solve (8), we need to segment the road pavement. In this aim, we apply a three step process:

1) Computation of a background model of the scene to filter all moving objects on the road surface

2) Rough manual segmentation of the road area to reduce the search area

3) Adaptive region growing in the background model restricted to the manually segmented area

Measuring the median intensity on each line of the pavement area then allows to obtain $I$, and to compute its inflection point $v_{i}$. A full description of this process is proposed in [11]. A sample result is given in Fig. 3. Based on (6), the surface covered by a pixel at the distance $d$ is expressed by:

$$
\Delta(d)=\frac{\lambda}{\left\lfloor v_{h}+\frac{\lambda}{d}\right\rfloor-v_{h}}-\frac{\lambda}{\left\lceil v_{h}+\frac{\lambda}{d}\right\rceil-v_{h}}
$$

where $\lfloor x\rfloor$ designates the whole part of $x$ and $\lceil x\rceil$ the integer greater than or equal to $x$. To fulfill the requirements expressed in [1], we have specified a video-surveillance system having the following characteristics:

\begin{tabular}{|c|c|c|c|c|c|}
\hline$D$ & $H$ & $f$ & $t_{p}$ & dim $_{y}$ & $\theta$ \\
\hline$[$ inch $]$ & {$[m]$} & {$[m m]$} & {$[\mu m]$} & {$[$ pix $]$} & {$[$ degree $]$} \\
\hline $1 / 2$ & 6 & 4.5 & 4.65 & 1360 & $28-29$ \\
\hline
\end{tabular}

Based on (9), we plotted the relative error on distance estimation with respect to the distance in Fig. 4. As one can see, the relative error at $400 \mathrm{~m}$ is smaller than $10 \%$. The theoretical accuracy of the proposed system is thus at least as good as the one provided by a road visibilitymeter.

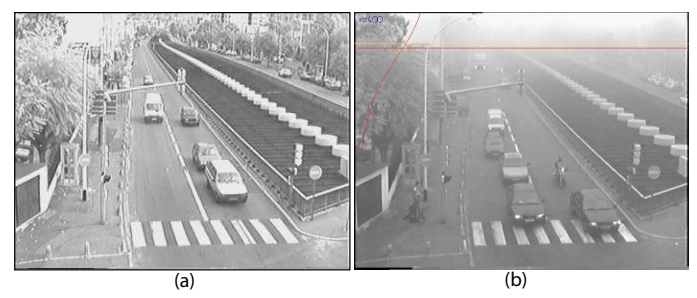

Figure 3. Daytime fog detection: (a) clear weather image; (b) foggy weather image. Meteorological visibility distance estimation is represented by the horizontal red line.

\section{B. In-vehicle Sensors}

1) Fog Lamps Status: Front fog lamps are intended to increase the illumination directed towards the road surface in conditions of poor visibility due to rain, fog, dust or snow [8], [17]. As such, they are often most effectively used in place of dipped-beam headlamps, reducing the glare back from fog. Based on [18], the usage of fog lamps during daytime increases with deterioration in weather conditions, with the usage reaching $50 \%$ of all installed fog lamps during moderate-to-heavy fog. This indicates that, during daytime, drivers adjust the usage of their lamps in response to weather conditions, which is not the case in night-time. Based on these statements, the status of the fog lights can be considered as a fog sensor in daytime.

2) Camera-based Sensor: The principle of this sensor is the same as in the roadside system. However, the implementation as well as the accuracy of the system are different. First, the camera is moving: the computation of the background model and the manual segmentation of the road surface are not possible, so we use the implementation proposed in [14]. Second, the expected accuracy of camera sensors is not as good as the roadside camera we specified previously. Indeed, the classical resolution of automotive cameras is smaller $(640 \times 480)$, as well as the mounting height of the sensor $(\approx 1.4 \mathrm{~m})$. To compare the accuracy of both solutions, we computed the relative error on visibility estimation with respect to the distance in Fig. 4.

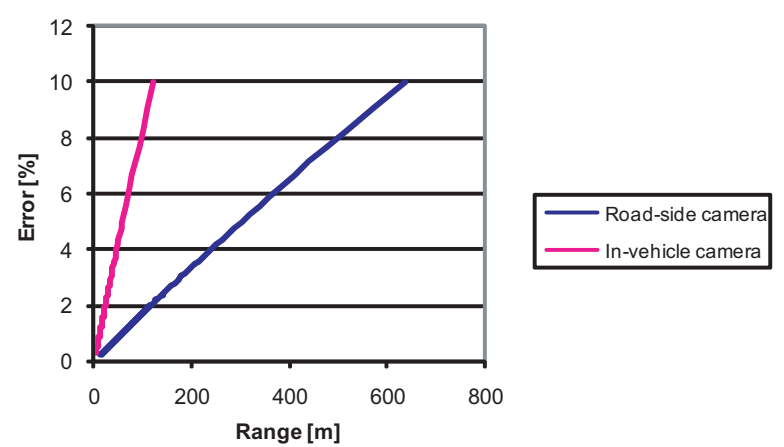

Figure 4. Relative error on visibility estimation with respect to the distance for the specified road-side camera and a classical in-vehicle camera. 


\section{SEnSORS COMBINATION FRAMEWORK}

\section{A. Objective}

In this section, we describe a general framework based on data fusion for dealing with uncertainty in the detection and characterization of critical environmental conditions such as dense fog. To illustrate our methodology, we use data issued from sensors (visibilitymeter, roadside camera, fog lamps status, in-vehicle camera). Each sensor is characterized by:

$$
\left\langle V^{j}, \varepsilon^{j}\right\rangle \text { with } j \in[1, \ldots, m]
$$

where $V^{j}$ is the visibility range measured by the $j^{\text {th }}$ sensor, $\varepsilon^{j}$ is the associated uncertainty (the sensor measurements are contaminated with noise) and $m$ is the number of sensors. At time $t$, we want to estimate the global visibility range $V_{t}^{g}$ of the studied area and $\varepsilon_{t}^{g}$ the global uncertainty on the visibility range of the studied area after fusion.

\section{B. Fog Detection Scenarios}

This section describes two scenarios for fog detection and density estimation. The first scenario examines fog detection using the fog lamps status of vehicles. In this scenario we suppose that the fog lamps are turned on manually by the driver in the presence of fog. The second scenario describes fog detection and density estimation by merging data from roadside and in-vehicle sensors.

1) First Scenario: In this scenario, we only consider fog lamps status as the source of information with an uncertainty $\varepsilon$. Fog lamps status is tracked in a specific detection area. After $n$ records, the probability of fog presence is estimated as following. We divide the possible outcomes from the vehicles into two complementary events, say $A$ (fog lamp status on) and $\bar{A}$ (fog lamp status off). We call an occurrence of the event $A$, a success $(S)$, and of $\bar{A}$, a failure $(F)$. We represent the probability of success for a single trial by $p$, and that of failure by $q=1-p$.

The number of sequences containing just $k S^{\prime} s$ is the number of combinations of $k$ items from $n, C_{k}^{n}$. Thus, to obtain $P(K=k)$, we have to sum the probabilities of the $C_{k}^{n}$ simple events which go to make up the event $K=k$. Therefore:

$$
\begin{aligned}
& P(K=k)=P_{k}=C_{k}^{n} p^{k} q^{n-k}= \\
& C_{k}^{n} p^{k}(1-p)^{n-k}, k=0,1, \ldots, n .
\end{aligned}
$$

Based on the paragraph IV-B1, setting $p=0.5$ allows to have an estimation of the probability of fog presence on the studied area. Unfortunately, we are not able to have a correct estimation of the visibility range.

2) Second Scenario: In this case, we consider that we have $p=m-n$ sensors. The sensors can be fixed (RSU sensor) or mobile (patrol vehicle or high-end vehicles equipped with fog sensors). The main advantage brought by this solution, compared to using only the fog lamps status, is the possibility of estimating the visibility range in different areas, as well as the uncertainty. We thus have at our disposal $p$ spatially distributed sensors. Each sensor has its own uncertainty due to its measurement principle. This was the topic of section IV. Then, at a specific location, where no sensor is present, the estimation of the visibility range depends on the different surrounding data sources. Since fog is a local phenomenon, the uncertainty strongly increases with the distance. The visibility distance should thus be expressed by the barycenter of the different sensor outputs, where the weights depend on the intrinsic sensor uncertainty and on the distance $d$ between the considered location and the sensor:

$$
V^{g} \propto \frac{\sum_{j=1}^{p} \frac{V_{j}}{\varepsilon^{j}(d)}}{\sum_{j=1}^{p} \frac{1}{\varepsilon^{j}(d)}} .
$$

The associated uncertainty is expressed by:

$$
\varepsilon^{g} \propto\left(\sum_{j=1}^{p} \frac{1}{\varepsilon^{j}(d)}\right)^{-1} \text {. }
$$

The dependency on the distance is very important. The influence of close-by sources must be important, whereas the influence of distant sources must be small. In this aim, we propose to use the probability distribution function introduced in [19] under the name of Smooth Exponential Family (SEF) $S_{\alpha, s}$ :

$$
\varepsilon^{j}(d)=\varepsilon_{\alpha, s}^{j}(d)=\underbrace{\varepsilon^{j}}_{j^{t h} \text { sensor uncertainty }}+\frac{1}{S_{\alpha, s}}
$$

where $S_{\alpha, s}=\frac{1}{s} e^{-\frac{1}{2} \phi_{\alpha}\left(\left(\frac{d}{s}\right)^{2}\right)} \cdot \phi_{\alpha}$ denotes the robust function where with $u=\left(\frac{d}{s}\right)^{2}, \phi_{\alpha}(u)=\frac{1}{\alpha}\left((1+u)^{\alpha}-1\right)$. Thanks to this approach, the influence of distant sources can be controlled by tuning the values of $s$ and $\alpha$.

\section{Data Fusion Model}

In the real world, the road network is equipped with fixed sensors, vehicles may have onboard sensors and the other vehicles are simply equipped with fog lamps. Based on (1114), we propose the following expression for the visibility range at a location of the road network, at time $t$ :

$$
\left\{\begin{array}{l}
V_{t}^{g}=\lambda \cdot \frac{\sum_{j=1}^{p} \frac{V_{j}}{\varepsilon_{\alpha, s}^{j}(d)}}{\sum_{j=1}^{p} \frac{1}{\varepsilon_{\alpha, s}^{j}(d)}}+\mu \cdot V_{t-1}^{g} \\
\text { with } \lambda+\mu=1
\end{array}\right.
$$

where the uncertainty is given by:

$$
\left\{\begin{array}{l}
\varepsilon_{t}^{g}=\beta \cdot\left(\sum_{j=1}^{p} \frac{1}{\varepsilon_{\alpha, s}^{j}(d)}\right)^{-1}+\gamma \cdot \frac{1}{P_{k}}+\eta \cdot \varepsilon_{t-1}^{g} \\
\text { with } \beta+\gamma+\eta=1
\end{array}\right.
$$

Then, a threshold $\varepsilon^{*}$ may be used on the uncertainty to discard least probable visibility range estimations. The value of this threshold can be chosen according to the criticality of the application. If the visibility estimation is used to warn the drivers about a potential fog bank ahead, a moderate threshold may be chosen. If it is used to compute a recommended speed, a low threshold should be chosen. 


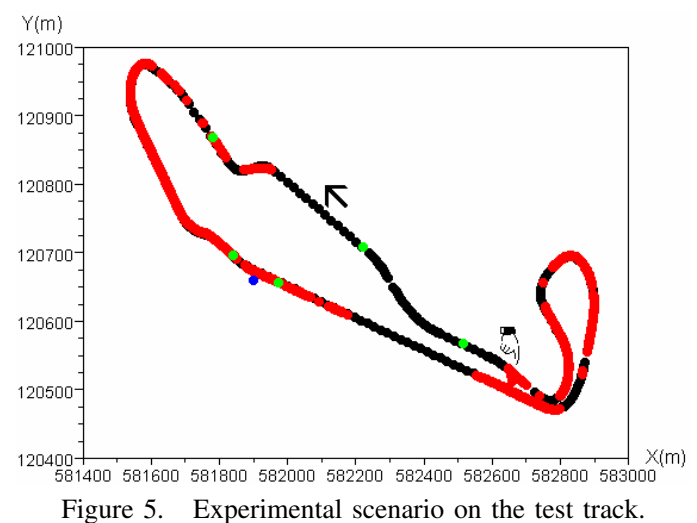

VI. EXPERIMENTAL EVALUATION

\section{A. Experimental Protocol}

To evaluate the proposed model, we have used real data recorded during a ride around a closed test track $(3.6 \mathrm{~km})$ in daytime fog. The test track is represented in Fig. 5. The starting point is represented by a small hand symbol and the driving direction by an arrow. The RSU is located at the blue point and is equipped with the camera described in section IV-A2. A car equipped with a high-precision differential GPS and a camera was used to record data (OBU data). Fog was detected at the black locations and was not detected at the red locations. We have selected five images taken at the green locations to estimate the visibility distance. It took approximately 5 minutes to record the data. Experimental data, including the estimations of visibility distance and the intrinsic sensor uncertainties $\epsilon$, are given in Tab. I. Assuming that fog density remained constant during this period, the rate of images where fog is detected can be assimilated with the status of fog lamps on a road section discussed in section IV-B1.

\section{B. Results}

We have set the parameters of the proposed data fusion framework as following. The scenario is static, so $\eta=0$ and $\mu=0$. Then, we have given the same weight to the fog lamp status and the sensors measurements to compute the uncertainty: $\beta=0.5$ and $\gamma=0.5$. Finally, we have set the parameters of the robust function $S_{\alpha, s}$ as following: $\alpha=0.5$ and $s=200$. We have computed the visibility

\begin{tabular}{|c|c|c|c|c|c|}
\hline Data type & Time issue & $\mathrm{X}(\mathrm{m})$ & $\mathrm{Y}(\mathrm{m})$ & $V_{\text {met }}$ & $\epsilon$ \\
\hline RSU & 0.00 .000 & 581900 & 120660 & 120 & 2.4 \\
OBU & 0.21 .627 & 582515 & 120568 & 125 & 15.6 \\
OBU & 0.45 .829 & 582222 & 120708 & 83 & 6.9 \\
OBU & 1.13 .405 & 581779 & 120869 & 71 & 5 \\
OBU & 2.14 .454 & 581839 & 120697 & 71 & 5 \\
OBU & 2.32 .673 & 581974 & 120657 & 125 & 15.6 \\
\hline
\end{tabular}

Table I

DATA USED FOR THE EXPERIMENTAL EVALUATION.

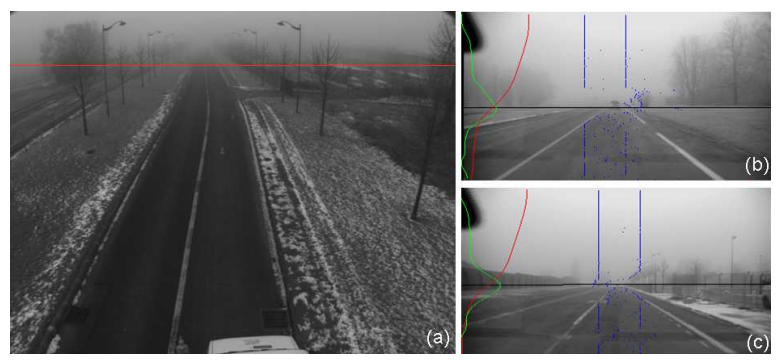

Figure 6. Sample images grabbed to estimate the visibility on the test track: (a) RSU camera $V_{\text {met }} \approx 120 \mathrm{~m}$; (b)(c) OBU camera: $V_{\text {met }} \approx 71$ $\mathrm{m}$ and $V_{\text {met }} \approx 125 \mathrm{~m}$.

map as well as the uncertainty map on the area around the test track by using the proposed data fusion framework. The results are given in Fig. 7. We have also extracted the profiles of visibility and uncertainty on the test track, given in Fig. 8. The probability of fog presence obtained with (11) is computed on a $4 \mathrm{~s}$ sliding window and explains the peaks in Fig. 8. It can be noticed that the interpolation of the visibility distance is quite smooth. It indicates that the fog density is higher in the North West part of the test track area and lower in the South East part of the test track area. This was checked visually thanks the video grabbed in the vehicle. In addition, no spots around the measurement points can be distinguished thanks to the use of the robust function $S_{\alpha, s}$. On the contrary, the uncertainty is very small around the measurement points, in particular near the RSU camera. We have computed that the total uncertainty on the test track is reduced by $10 \%$ thanks to the RSU camera. Finally, the mean visibility estimated thanks to all the images grabbed in the vehicle is around $100 \mathrm{~m}$, which is in agreement with the visibility map shown in Fig. 7.

\section{Conclusion And Perspectives}

In this paper, a data fusion framework based on the combination of roadside sensors and in-vehicle devices is presented aiming at taking the advantages of both fixed sensors and mobile sensors and at proposing an extensive detection and estimation of the fog density. It enables to obtain a homogeneous view on the visibility range on the entire network and allows the road operator to decide if mandatory speed reductions should be triggered. This framework takes place in the architecture of the SAFESPOT project, which was recalled. We presented the different data sources for meteorological visibility that we have at our disposal, in particular our camera-based approach. Simulated results illustrate the efficiency of the proposed solution. However, the parameters of our data fusion framework have to be set with respect to the dynamics of a fog event. In this aim, more tests on test tracks as well as on open roads are foreseen in the fourth year of the SAFESPOT project. 


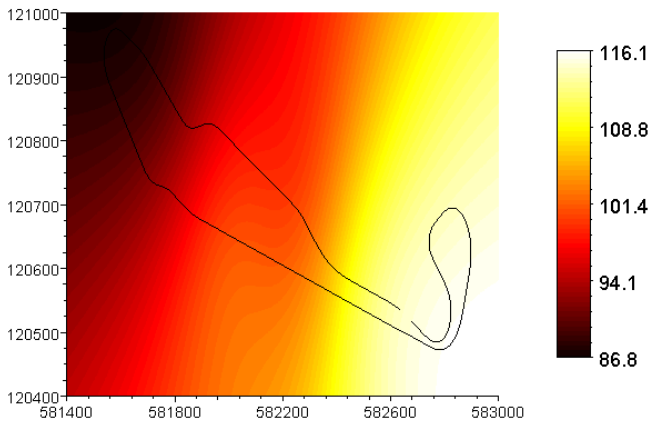

(a)

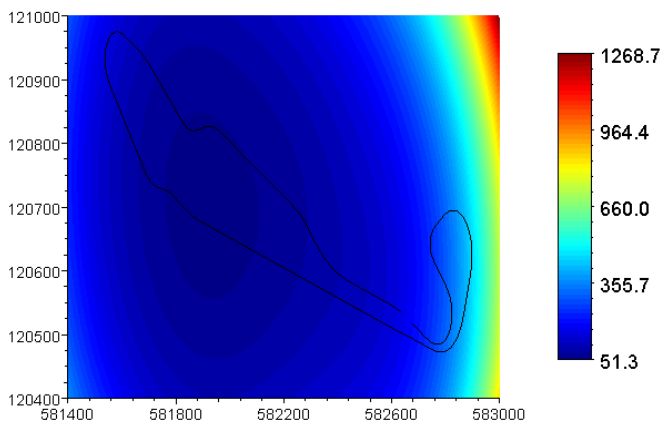

(b)

Figure 7. Results: (a) Map of meteorological visibility distance extrapolated on the area of the test track; (b) Map of uncertainty on the meteorological visibility distance extrapolated on the area of the test track.

\section{ACKNOWLEDGMENT}

This work is supported by the European Integrated Project SAFESPOT (IST-4-026963-IP).

\section{REFERENCES}

[1] AFNOR. Road meteorology - gathering of meteorological and road data - terminology. NF P 99-320, April 1998.

[2] C. Bartels. SAFESPOT Local Dynamic Maps - virtual world for safety applications. In ITS World Congress, Beijing, China, 2007.

[3] C. Boussard, N. Hautière, and B. d'Andréa Novel. Vehicle dynamics estimation for camera-based visibility distance estimation. In IEEE/RSJ International Conference on Intelligent RObots and Systems, 2008.

[4] R. Brignolo, L. Andreone, and G. Burzio. The SAFESPOT Integrated Project: Co-operative systems for road safety. In Transport Research Arena, Göteborg, Sweden, 2006.

[5] C. Bush and E. Debes. Wavelet transform for analyzing fog visibility. IEEE Intell Syst, 13(6):66-71, November/December 1998.

[6] CIE. International Lighting Vocabulary. Number 17.4. 1987.

[7] J. Crosby. Visibility sensor accuracy: what's realistic? In 12th Symposium on Meteorological Observations and Instrumentation, 2003.

[8] ECE R48. Front fog lamp, October 1995.

[9] T. Hagiwara, Y. Ota, Y. Kaneda, Y. Nagata, and K. Araki. A method of processing CCTV digital images for poor visibility identification. Transport Res Rec, 1973:95-104, 2007.

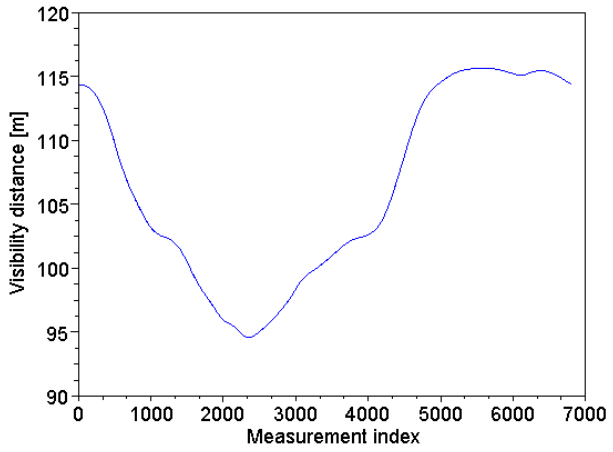

(a)

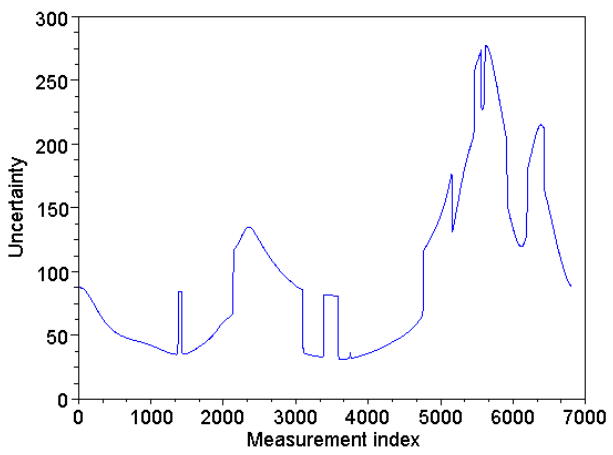

(b)

Figure 8. Results: (a) Profile of meteorological visibility distance computed on the test track; (b) Profile of uncertainty on the meteorological visibility distance computed on the test track.

[10] R. Hallowell, M. Matthews, and P. Pisano. An automated visibility detection algorithm utilizing camera imagery. In AMS Annual Meeting, 2007.

[11] N. Hautière, E. Bigorgne, and D. Aubert. Visibility range monitoring through use of a roadside camera. In IEEE Intelligent Vehicles Symposium, 2008.

[12] N. Hautière, R. Labayrade, and D. Aubert. Estimation of the visibility distance by stereovision: a generic approach. IEICE T Inf Syst, E89-D(7):2084-2091, July 2006.

[13] N. Hautière, R. Labayrade, and D. Aubert. Real-time disparity contrast combination for onboard estimation of the visibility distance. IEEE T Intell Transp, 7(2):201-212, June 2006.

[14] N. Hautière, J.-P. Tarel, J. Lavenant, and D. Aubert. Automatic fog detection and estimation of visibility distance through use of an onboard camera. Mach Vision Appl, 17(1):8-20, 2006.

[15] A. Lagorio, E. Grosso, and M. Tistarelli. Automatic detection of adverse weather conditions in traffic scenes. In IEEE International Conference on Advanced Video and Signal based Surveillance, pages 273-279, 2008.

[16] W. Middleton. Vision through the atmosphere. University of Toronto Press, 1952.

[17] SAE Standard J583. Front fog lamp, September 2005.

[18] M. Sivak, M. Flannagan, E. Traube, H. Hashimoto, and S. Kojima. Fog lamps: Frequency of installation and nature of use. SAE Technical Paper 970657, 1997.

[19] J.-P. Tarel, S.-S. Ieng, and P. Charbonnier. Using robust estimation algorithms for tracking explicit curves. In European Conference on Computer Vision, 2002. 